\title{
Evaluación de la actividad antiinflamatoria de una crema a partir del extracto purificado de Baccharis Tricuneata (L.f.) Pers. "taya"
}

\section{Evaluation of the antiinflammatory activity of a cream from purified extract from Baccharis Tricuneata (L. f.) Pers. "Taya"}

\author{
Marlon Díaz ${ }^{1}$, John Conde ${ }^{1}$, Pedro Félix ${ }^{1}$, Sandra Ramírez¹, Roly Vicuña ${ }^{1}$ \\ ${ }^{1}$ Asociación Científica de Investigación Farmacéutica - Facultad de Farmacia y Bioquímica- Universidad \\ Nacional "San Luis Gonzaga" de Ica.
}

\section{RESUMEN}

El género Baccharis es una de las más importantes fuentes de productos en la medicina natural, se distribuyen en el Norte y Sur de América y en nuestro país en las zonas alto andinas, despertando el interés por su uso etnofarmacológico. La especie Baccharis tricuneata (L.f.) pers. "taya", es usada en la medicina tradicional en la terapia antiinflamatoria en forma de emplastos.

El extracto acetato de etilo se obtuvo a partir del extracto etanólico por sucesivos fraccionamientos utilizando solventes polaridad creciente. Se realizó la identificación de flavonoides en los extractos mostrando mayor presencia en el extracto de acetato de etilo.

Se formuló y evaluó la actividad antiinflamatoria de una crema elaborada a partir del extracto de acetato de etilo a varias concentraciones. La evaluación farmacológica se realizó por el método de Edema Auricular Inducido por Aceite de Croton.

La crema al $30 \%$ mostró actividad antiinflamatoria del $71.43 \%$. Se atribuye a los flavonoides la actividad antiinflamatoria encontrada ya que existen reportes de su gran poder de estabilizar membranas y los radicales oxidantes.

Descriptores: etnofarmacológico, Baccharis tricuneata, actividad antiinflamatoria.

\section{ABSTRACT}

The genus Baccharis is one of the most important sources of products in the natural medicine, are distributed in the North and South America and in our country in the high Andean areas, arousing the interest of their usage etnofarmacologico. The species Baccharis tricuneata (L. f.) Pers. "Taya", is used in traditional medicine in the antiinflammatory therapy in the form of poultices.

The ethyl acetate extract was obtained from the ethanolic extract by successive fragmentations using solvents increasing polarity. It carried out the identification of flavonoids in the extracts showing greater presence in the ethyl acetate extract. It was formulated and assessed the antiinflammatory activity of an ointment prepared from the extract of ethyl acetate to various concentrations. Pharmacological evaluation was performed by the method of ear edema induced by Croton Oil.

The cream to the $30 \%$ showed antiinflammatory activity of $71.43 \%$. It is attributed to the flavonoids the antiinflammatory activity found that there are already reports of his great power to stabilize membranes and oxidizing radicals.

Keywords: ethnopharmacological, Baccharis tricuneata, anti-inflammatory activity.

\section{INTRODUCCIÓN}

La piel constituye el $16 \%$ del peso del cuerpo. Por su carácter de cobertura/envoltura externa corporal está especialmente expuesta a traumatismos, que dan origen a un proceso inflamatorio. La inflamación es una de las respuestas fisiopatológicas fundamentales con las que el organismo se defiende frente agresiones producidas por gran variedad de estímulos ${ }^{1}$, liberando sustancias mediadoras de la inflamación como histaminas que ocasionan daño celular $^{2,3}$. Existen diversos mecanismos por el cual se puede tratar un proceso inflamatorio. El uso de plantas medicinales ha demostrado tener estos efectos por poseer propiedades antiinflamatorias de metabolitos secundarios como los flavonoides, antocianidinas, cumarinas, taninos, etc ${ }^{4,5}$. El Género Baccharis es uno de los más importantes de la familia Asteraceae, ha despertado en interés por su 
uso etnofarmacológico, farmacológico y fitoquímico ${ }^{6}$, se distribuyen en todo el Norte y Sur de América y en nuestro país en las zonas alto andinas en donde es utilizada en el tratamiento de heridas, ulceras, fiebre, enfermedades Gastrointestinales, diuréticos, analgésicos, tratamientos de diabetes, antibiótico, y mordedura de serpiente ${ }^{7}$. La especie Baccharis tricuneata (L.f) Pers. popularmente llamada "taya" es utilizada como emplastos de hojas y en el tratamiento de infecciones de piel, inflamación y diabetes. Estudios realizados demostraron la presencia y elucidación de metabolitos activos, así como la determinación de la actividad antiinflamatoria y antibacteriana. El propósito del estudio es determinar la actividad antiinflamatoria de la crema obtenida del extracto con mayor actividad utilizando el método de edema auricular inducido por aceite de Croton y proponer un mecanismo de los metabolitos responsables de la actividad. Se eligió elaborar una crema con la finalidad de incorporar el extracto activo, utilizando una emulsión o/w, fácilmente lavable y sensación de frescura, mostrando cualidades organolépticas características, además sus valores de $\mathrm{pH}$ se encuentran dentro de los márgenes de $\mathrm{pH}$ de la piel humana $(5.0-6.5)$.

\section{PARTE EXPERIMENTAL}

Tipo de estudio: Experimental de diseño cuasi experimental $^{8,9}$

\section{Material y equipos}

\section{. Material Biológico:}

- Tallos de Baccharis tricuneata (L.f.) Pers "taya"

Ratones Albinos cepa Balb/c 53 hembras de 25-30 gr. Procedentes del "Centro Nacional de Productos Biológicos" del Instituto Nacional de Salud de Chorrillos- Lima.

\section{. Material de Laboratorio:}

Reactivos: Etanol, acetato de etilo, eter de petroleo, dicloruro de metileno, agua destilada, twin 80 , vaselina solida, vaselina líquida, trietanolamina, ácido esteárico.

Equipos: Balanza analítica marca BOECO modelo BPB 31, Rotavapor marca HEIDOLPH modelo LABORATORA 4000), Micropipeta modelo Brand marca TRANSFERPETTE. Balanza de precisión SARTORIUS.

\section{Métodos y Procedimientos}

\subsection{Ensayo fitoquímico}

\subsubsection{Recolección y secado de la planta}

La especie vegetal fue recolectada en el pueblo de Ocaña, provincia de Lucanas, departamento de Ayacucho, en el mes de febrero del 2006 a una altitud de 2660 m.s.n.m, secado al aire libre en un lugar seco y sin presencia de rayos solares por un periodo de 21 días, los tallos se molieron en un molino de manual.

\subsubsection{Clasificación taxonómica:}

La muestra vegetal completa ha sido estudiada y clasificada como: Baccharis tricuneata (L.f.) Pers. Y tiene la siguiente posición taxonómica, según el Sistema de Clasificación de Cronquist (1988).

\section{DIVISION: MAGNOLIOPHYTA \\ CLASE: MAGNOLIOPSIDA \\ ORDEN: ASTERALES \\ FAMILIA: ASTERACEAE \\ GENERO: Baccharis \\ ESPECIE: Baccharis tricuneata (L. f.) Pers. \\ Nombre vulgar: "taya" \\ Determinada por: Mg Joaquina Albán C. museo de Historia Natural.}

\subsubsection{Obtención del extracto etanólico:}

Un kilogramo de tallos secos y molidos de la especie fueron sometidos a extracción en reflujo con etanol de $96^{\circ}$ hasta agotamiento, la evaporación del disolvente se realizó en un evaporador rotatorio a presión reducida modelo HEIDOLPH LABORATORA 4000 hasta sequedad a una temperatura de $40^{\circ} \mathrm{C}$. Obteniendo $350 \mathrm{~g}$ de extracto seco de color marrón oscuro.

\subsubsection{Obtención de la fracción de acetato de etilo:}

El extracto etanólico se suspendió en una mezcla de éter de petróleo y agua (1:1) en un embudo de decantación, luego se utilizó solventes de polaridad creciente (éter de petróleo, diclorometano, acetato de etilo, agua), a fin de tener fracciones de distinta polaridad. 
Flujograma Fig. 1. Partición del extracto etanólico con solventes de deferente polaridad.

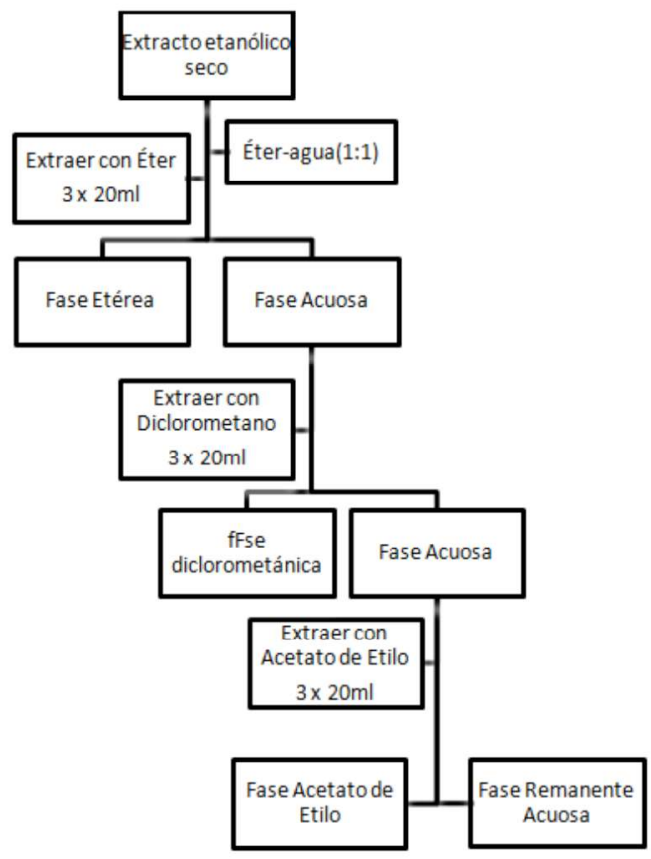

\subsection{Pre-formulación de cremas}

2.2.1. Ensayo de solubilidad de la fracción de Acetato de Etilo.

En una primera etapa se realizó un ensayo se solubilidad del extracto en excipientes solubles que se emplean en el proceso de elaboración de la crema. Esta prueba se realizó de la siguiente manera:

Pesar $0.1 \mathrm{~g}$ de la fracción de Acetato de Etilo y disolver en $2 \mathrm{~mL}$ del excipiente utilizado en un tubo de ensayo.

\section{TABLA N 1: SOLUBILIDAD DE LA FRACCIÓN DE ACETATO DE ETILO EN LOS EXCIPIENTES.}

\begin{tabular}{lllll} 
& Agua & Alcohol & Glicerina & Vaselina \\
Fracción de & + & +++ & ++ & - \\
acetato de Etilo & & & & \\
\hline Fuente datos de los autores- Laboratorio de Tecnología Farmacéutica de la \\
Facultad de Farmacia y Bioquímica de la U.N.ICA
\end{tabular}

El ensayo de solubilidad sirve de criterio para la elección de excipiente adecuado para cada extracto, permitiendo su incorporación durante la elaboración de la crema.

\subsubsection{Fórmula para la elaboración de la crema:}

Luego del ensayo preliminar se selecciona el excipiente con mayor solubilidad para la fracción de Acetato de Etilo.

Siendo esta la formula la siguiente:
Ácido esteárico

$16 \mathrm{~g}$

Trietanolamina (TEA)

Vaselina sólida

Extracto de acetato

Alcohol

Agua c.s.p.

$$
\begin{array}{r}
1 \mathrm{ml} \\
4 \mathrm{~g} \\
\mathrm{Xg} \\
1 \mathrm{ml} \\
100 \mathrm{ml}
\end{array}
$$

\section{Procedimiento}

Pesar cada uno de los componentes a utilizar, En un vaso de precipitado verter el ácido esteárico y adicionar vaselina sólida, fundir con ayuda de calor y llevar a ebullición, esto constituye la mezcla 1. Simultáneamente en otro vaso de precipitado verter TEA y luego agua, llevar a calor, hasta ebullición. Esto constituye la mezcla 2. Incorporar la mezcla 1 en la mezcla 2 con agitación constante y vigorosidad, hasta uniformidad, retirar del calor y dejar enfriar. Con la ayuda de un mortero disolver el extracto seco de acetato de etilo con el mínimo volumen de etanol de $96^{\circ}$, e incorporar poco a poco la base obtenida anteriormente con agitación contante, hasta observar uniformidad. Colocar las fórmulas en potes debidamente etiquetados. Luego medir características organolépticas como: el aspecto, textura, color, olor.

\section{Determinación de pH:}

Se calibró el pH-metro digital (HANNA instruments) con dos soluciones buffer de $\mathrm{pH} 7$ y 4 .

Se peso $1 \mathrm{~g}$ de la crema y se colocó en un vaso de precipitado con $10 \mathrm{~mL}$ de agua destilada, disolver y colocar el electrodo para medir el $\mathrm{pH}$ correspondiente.

\subsection{Ensayo farmacológico}

\subsubsection{Método de edema auricular inducido por Aceite de Croton (Croton tigilum L.) ${ }^{10}$}

Se basa en la aplicación tópica del agente flogógeno (aceite de croton) en el cual actúa como un irritante vascular en el pabellón auditivo del ratón con filtración de leucocitos polimorfonucleares y edema intercelular produciendo una respuesta inflamatoria.

\subsubsection{Animales de experimentación}


Se forman lotes de 5 ratones albinos hembras de aproximadamente $25-30 \mathrm{~g}$ de peso corporal. Se les somete a un ayuno previo de 12 horas antes de iniciar el ensayo, siendo distribuidos de forma aleatoria en los grupos de estudio.

\subsubsection{Muestras en estudio}

Las cremas elaboradas al $1,5,10$ y $30 \%$

Se utilizó un fármaco de referencia Betametasona en crema al $0.05 \%$.

\subsubsection{Descripción de la técnica}

Se prepara una disolución al $5 \%$ de aceite de Croton en etanol de $96^{\circ}$, se administra $20 \mu \mathrm{L}$ del agente irritante en la oreja derecha del ratón con una micropipeta automática (Humapette), depositando 10 $\mu \mathrm{L}$ en cada cara (externa e interna) del pabellón, a la oreja izquierda solo se aplica el vehículo. Los grupos tratados recibieron inmediatamente después del aceite de Croton por vía tópica $20 \mu \mathrm{L}$ en cada cara de oreja derecha) de las primeras muestras en estudio. Respecto a las cremas y al fármaco de referencia se administraron $20 \mathrm{mg}$ aproximadamente en cada cara del pabellón auricular hasta cubrir toda la superficie de manera uniforme. Para ambas muestras de estudio el control positivo fue Betametasona al $0.05 \%$.

La oreja izquierda sólo recibe el vehículo.

Transcurrida cuatro horas después de la aplicación, se sacrificaron a los animales por dislocación cervical y se procedió a cortar con un sacabocado porciones circulares de $7 \mathrm{~mm}$ de la oreja inflamada y no inflamada, pesándolas inmediatamente. La actividad antiinflamatoria se calculó mediante las siguientes fórmulas:

Porcentaje de inflamación $=\underline{T \times 100}-100$

\section{ST}

Donde $\mathbf{T}$ corresponde a la media de los pesos de las orejas tratadas y ST a la media de los pesos de las orejas sin tratar.

Porcentaje de inhibición $=\underline{C-T} \times 100$
Donde C corresponde el porcentaje de inflamación del grupo control y T el porcentaje de inflamación del grupo en estudio o problema.

\section{RESULTADOS Y DISCUSIÓN}

TABLA N 2: CUALIDADES ORGANOLÉPTICAS Y FÍSICAS QUE PRESENTAN LAS CREMAS AL 1, 5 , $10,30 \%$.

\begin{tabular}{ccccc}
\hline & \multicolumn{4}{c}{ Cremas } \\
\cline { 2 - 5 } & $\mathbf{1 \%}$ & $\mathbf{5 \%}$ & $\mathbf{1 0 \%}$ & $\mathbf{3 0 \%}$ \\
\hline Aspecto & Brillante & Brillante & Opaco & Opaco \\
\hline Textura & Suave al tacto & Suave al tacto & Suave al tacto & Suave al tacto \\
\hline Color & Crema claro & Crema & Crema oscuro & Marrón claro \\
\hline Olor & Característico & Característico & Característico & Característico \\
\hline $\mathbf{p H} / \mathbf{2 0}{ }^{\circ} \mathrm{C}$ & 6.41 & 6.09 & 6.32 & 5.96 \\
\hline
\end{tabular}

Fuente datos de los autores- Laboratorio de Control de Calidad de la Facultad de Farmacia y Bioquímica de la U.N.ICA

\section{TABLA N ${ }^{\circ}$ 3: MEDIA DE PESOS DE OREJAS DE LOS RATONES.}

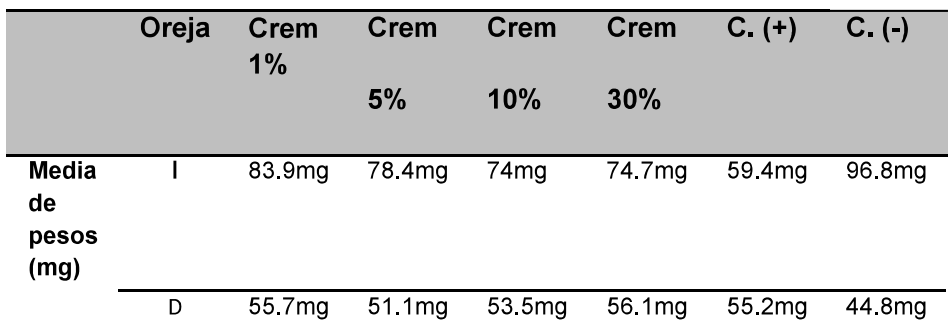

Fuente datos de los autores- Laboratorio de Farmacología de la Facultad de Farmacia y Bioquímica de la U.N.ICA

\section{TABLA N 4: PORCENTAJE DE INFLACIÓN E INHIBICIÓN DE LA INFLAMACIÓN DE LAS CREMAS AL $1,5,10,30 \%$.}

\begin{tabular}{lccc}
\hline & $\begin{array}{l}\text { Peso de oreja } \\
\text { inflamada en } \\
\text { mg }\end{array}$ & $\begin{array}{l}\% \text { de } \\
\text { Inflamación }\end{array}$ & $\begin{array}{l}\% \text { de } \\
\text { inhibición } \\
\text { Inflamatoria }\end{array}$ \\
\hline CONTROL (-) & $52 \mathrm{mg}$ & $116.07 \%$ & \\
\hline CONTROL (+) & $4.2 \mathrm{mg}$ & $7.61 \%$ & $53.44 \%$ \\
\hline Crema 1\% & $28.2 \mathrm{mg}$ & $50.62 \%$ & $60.72 \%$ \\
\hline Crema 5\% & $27.3 \mathrm{mg}$ & $45.59 \%$ & $66.99 \%$ \\
\hline Crema 10\% & $20.5 \mathrm{mg}$ & $38.31 \%$ & $71.43 \%$ \\
\hline Crema 30\% & $18.6 \mathrm{mg}$ & $33.15 \%$ & \\
\hline $\begin{array}{l}\text { Fuente datos de los autores- Laboratorio de Farmacología de la Facultad de } \\
\text { Farmacia y Bioquímica de la U.N./CA }\end{array}$
\end{tabular}




\section{GRÁFICA N 1 CONCENTRACIÓN- EFECTO DE INHIBICIÓN DE LA INFLAMACIÓN DE LAS \\ CREMAS AL 1, 5, 10, 30\%.}

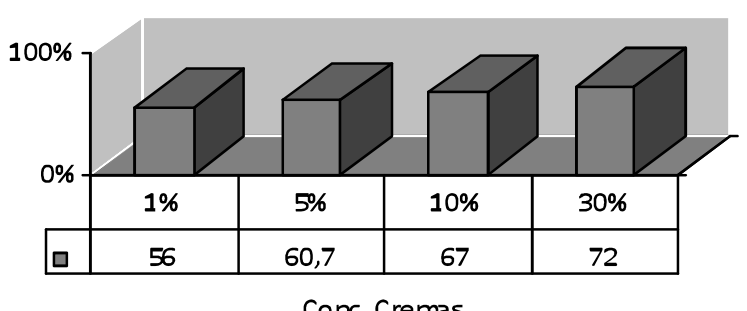

La inflamación es una de las respuestas fisiopatológicas fundamentales con las que el organismo se defiende frente agresiones producidas por gran variedad de estímulos ${ }^{1}$, liberando sustancias como histaminas que ocasionan daño celular $^{2,3}$. Existen diversos mecanismos por el cual se puede tratar un proceso inflamatorio. El uso de plantas medicinales ha demostrado tener estos efectos por poseer propiedades antiinflamatorias de los flavonoides, cumarinas, taninos, etc ${ }^{4,5}$ Que se deben principalmente a su acción antioxidante y a su habilidad de actuar contra las histaminas y otros mediadores de inflamación, como las prostaglandinas y los leucotrienos. En investigaciones anteriores se demostró que el extracto de Baccharis tricuneata posee diversos metabolitos secundarios además de la actividad antiinflamatoria y antibacteriana.

Se eligió elaborar una crema con la finalidad de incorporar el extracto activo tomando como base conocimientos farmacocinéticos y de tecnología farmacéutica, utilizando una emulsión o/w, fácilmente lavable y sensación de frescura, mostrando cualidades organolépticas características, además sus valores de $\mathrm{pH}$ se encuentran dentro de los márgenes de $\mathrm{pH}$ de la piel humana $(5.0-6.5)$.

La crema elaborada a diferentes concentraciones mostro significativa actividad antiinflamatoria desde la mínima concentración al $1 \%$ de $56.38 \%$ mostrando mayor efectividad conforme aumentaban la concentración del extracto. Se les atribuye la actividad a los metabolitos secundarios presentes en el extracto como los flavonoides por su reconocido poder antioxidante por un mecanismo de estabilización de membranas y reconociendo que la crema elaborada es la idónea para la aplicación del extracto.
1. La formulación de las cremas a diferentes concentraciones elaboradas a partir del extracto etanólico purificado de Baccharis tricuneata presentan significativa actividad antiinflamatoria.

2. Se obtuvo la fracción de acetato de etilo, y con esta se formuló la crema a concentraciones de $1,5,10$ y $30 \%$.

3. Las cremas mostraron características peculiares como olor característico, la textura suave al tacto, el aspecto de las cremas al $1 \%$ y $5 \%$ brillante, mientras que el aspecto de las cremas al $10 \%$ y $30 \%$ fue opaco. El pH a $20^{\circ} \mathrm{C}$ de las cremas al $1,5,10$ y $30 \%$ fue de $6.41,6.09,6.32$ y 5.96, por lo que se puede decir que son actas para la aplicación en piel humana.

4. La actividad antiinflamatoria de la crema al $1 \%$ fue de $56.38 \%$, la crema al $5 \%$ de $60.72 \%$, la crema al $10 \%$ de $66.99 \%$ y la de $30 \%$ fue de $71.43 \%$.

5. El mecanismo de acción puede estar relacionado con la inhibición de la inflamación provocada por las sustancias oxidantes, donde los metabolitos secundarios de estructuras fenólicas como los flavonoides presentes en el gel, por su gran poder de estabilizar membranas y atenuar el efecto de moléculas oxidantes como los formadas en un proceso inflamación

\section{AGRADECIMIENTOS}

Agrademos a la Asociación Científica de Investigación Farmacéutica ACIF, por la oportunidad de desarrollar esta investigación, a nuestros asesores como la Dra. Haydee Chávez Orellana, Q.F Carmela Ferreyra Paredes, Q.F Luisa Revatta Salas, Mg. Q.F Manuel Valle Campos y de manera muy especial a nuestros padres y amigos.

\section{REFERENCIAS}

[1] Jesús Flores, Farmacología Humana, $5^{\mathrm{a}}$ Edición, Editorial El Sevier Masson (2008) 426.

[2] Beltram G. Katzung, Farmacología Básica y Clinica, $11^{\text {a }}$ Edición, Editorial Mc Graw Hill (2010) 621

[3] Goodman Gilman A., Hardman JG y Limbird LE. Las Bases Farmacológicas de la Terapéutica. $10^{a}$ edición. Editorial Mc GrawHill Interamericana. 2005 
[4] Nikolai Sharapin. Fundamentos de Tecnología de Productos fitoterapéuticos. Publicación del Convenio Andrés Bello y el Programa Iberoamericano de Ciencia y tecnología para el Desarrollo CYTED. Santafé de Bogotá , D.C., Colombia. 2000.

[5] Boletín Latinoamericano y del Caribe de Plantas Medicinales y Aromáticas. México. Volumen 8, Número 1, Enero de 2009

[6] Abad MJ y Bermejo P.

Baccharis(Compositae): a review update. Dept. of Pharma, faculty of pharmacy, University Complutense. Rev. 2007-76-80.

[7] Brack A, Diccionario Enciclopedia de Plantas Medicinales Útiles del Perú. Cuzco PerúEditorial Centro de Estudios Regionales Andinos Bartolomé de las CasasPNUD.1999.
[8] Carrasco Díaz S., Metodología de la Investigación Científica.1ra Edición. Editorial San Marcos. 2009 pag 58-70.

[9] Canales FH, Alvarado EL y Pineda EB. Metodología de la Investigación Manual para el desarrollo de personal de salud. Editorial Limusa noriega editores. Publicación PASCCAP N¹6. México. 2005

[10] Cytec. Programa interamericano de Ciencia y Tecnología para el Desarrollo, Marzo 1995.

E-mail: marlondeval@hotmail.com 